\title{
Using Model Calibration to Improve Urban Modeling
}

\author{
Louis Leroy $^{1}$, Samuel Letellier-Duchesne ${ }^{1}$, Michaël Kummert ${ }^{1}$ \\ ${ }^{1}$ Polytechnique Montréal, Montreal, Canada
}

\begin{abstract}
Urban Energy Modeling (UBEM) often relies on "typical" buildings, called archetypes, to represent the modeled building stock. These archetypes include basic assumptions on parameters that play a significant role on energy use, such as thermal characteristics of the building envelope and occupancy-related parameters (e.g. setpoints and internal gains). These parameters are then combined to a geometrical approximation of the buildings to create a complete Building Performance Simulation (BPS) model. The increasing availability of measured data opens interesting perspectives for calibration but also new challenges. This paper compares different urbanlevel models of selected buildings (residential and commercial) with measured data. Detailed, calibrated models are also considered in the comparison, to represent "best achievable models". Results show that calibrated parameters from detailed models do not necessarily improve the accuracy of urban-level models. The simplifications in the selected urban-level archetypes have a significant impact in some cases, but a reasonable accuracy can be achieved for annual energy use. The dynamic profile, which would be required for energy flexibility and grid interaction studies, and for district energy assessment, is poorly represented by the selected urban-level models.
\end{abstract}

\section{Introduction}

Urban Building Energy Modeling (UBEM) is increasingly the focus of discussion and research inside energy modeling circles. The latest developments have enabled energy assessments of neighborhoods and even whole cities with acceptable computation time, allowing UBEM to become a relevant planning and decisionmaking tool in the areas of urban development, building retrofit policy making and energy systems optimization. Early UBEM studies often focused on analyzing the impact of building retrofits or quantifying heat rejection from building blocks, e.g. to assess their impact on the urban heat island effect (Heiple and Sailor 2008). Recent studies keep a strong focus on building retrofitting scenarios, but increasingly also aim at assessing the potential of district energy systems. For example, Sokol et al. (2017) created models to study building retrofits and district energy potential in Cambridge, MA. Building simulationists typically favor "bottom-up" approaches over "top-down" approaches for building stock modeling, as they allow detailed diagnostics and predictive studies at various scales, from country/region to specific neighborhoods (Reinhart and Cerezo Davila 2016; Sokol et al. 2017).

With a bottom-up modeling approach, modelers rely on extensive datasets that characterize each and every building inside the study area. To reduce the datapreparation task, several authors have proposed different approaches. Filogamo et al. (2014) used typological buildings (i.e. archetypes) classified by building age (7 categories), geometric properties (Window-to-Wall Ration [WWR], floor area, volume, etc.), thermo-physical properties (loss coefficient, thermal capacity), HVAC systems, and climate zones (using the Degree-Day method). While Kohler et al. (2002) concluded that studies are limited by the absence of reliable statistical data, Mata et al. (2014) created 593 archetypes solely based on building age, heating system type and climate zone, and compared annual final energy demand by enduse with statistical data from government surveys and individual building utility bills. Their results show the advantage of using building archetypes to compare enduse consumption with statistics from aggregated and areanormalized consumption values. Cerezo et al. (2015) remarked that with this type of approach, the estimated energy required for the whole building stock is accurate within 5 to $20 \%$, whereas this error can go up to $99 \%$ for individual buildings. One of the solutions proposed by Samuelson et al. (2015) is to calibrate the archetypes to further improve the accuracy of the results.

UMI (Urban Modeling Interface (Reinhart 2019)) is one of the few available state-of-the-art UBEM environments. The program is implemented as a Rhinoceros-based plugin, relying on the well-known 3D modeling environment (Robert McNeel \& Associates 2019) for geometrical aspects and implementing "templates" which contains non-geometric properties of the archetypes. Energy modelers are used to seeing archetypes as full 3D models with specific zoning schemes, orientations and façade elements (e.g. windows), and even a full description of the HVAC system (see for example (US DOE - BTO 2018)). UMI defines archetypes in a slightly different way. Archetypes defined in the template contain only non-geometric properties. Building shapes are created inside Rhino (for example using " $2.5 \mathrm{D}$ " extrusions from building footprints) and WWRs are specified by the user. This geometrical information is then 
combined with the "template" information into an EnergyPlus model to solve the energy simulation (U.S. Department of Energy's Building Technologies Office 2019). The developers of this tool have proposed an original approach to handle thermal zoning: UMI creates distinct smaller finite volumes, called "shoeboxes"-at least 1 per orientation-depending on the chosen template, WWR and solar incidence. The shoeboxes are then simulated independently, and the building-level energy performance data is obtained by a weighted sum of the shoeboxes comprising that building. Systems are not explicitly modeled, and constant COPs are used instead. Furthermore, UMI is designed as an Application Programing Interface (API) to help the development of complementary modules. For example, the investigation of district-level energy supply scenarios is developed in work by Letellier-Duchesne et al. (2018).

The increasing availability of measured data (e.g. from smart meters) offers new avenues for model calibration at different time scales, from yearly Energy Use Intensity (EUI) to hourly electricity use. But significant challenges remain, especially with high-uncertainty parameters such as schedules or occupancy patterns (Heiple and Sailor 2008), and the relationship between detailed calibration of individual buildings and neighborhood-level studies is an unexplored field that this paper proposes to investigate.

\section{Objectives}

The work presented in this paper aims at comparing different urban-level models of selected buildings with an increasing level of calibration to the local context. Without losing the simplified nature of urban-level models, is it possible to achieve a better accuracy thanks to measured energy data? And how far would "refined" urban-level energy models be from detailed, calibrated energy models? The paper focuses on a few commercial and residential buildings for which hourly measured energy data are available. Successive refinements to the archetype characterizations are implemented, and the building-level energy use is compared to measured data. The results are also compared to detailed and calibrated models, which were developed to estimate the energy end-use breakdown and to obtain a representation of "best achievable model".

\section{Methodology}

\section{Selected buildings}

The paper focuses on mixed-use neighborhoods in Montreal, QC. We have selected 4 buildings for which hourly energy data is available: one medium-sized commercial building (offices), and three single-family residential buildings (row townhouses or condominium units). The 3 residential buildings have the following characteristics:

- House 1: row house, $188 \mathrm{~m}^{2}$ living area, 2 floors above ground, typical recent construction (relatively recent retrofit), occupied by a family of 4 .

- House 2: housing unit within a row house, ground floor with an unoccupied (and unheated) basement, 94 $\mathrm{m}^{2}$, occupied by a family of 4, typical older construction (no energy retrofit). Another housing unit is located above.

- House 3: row house with 2 floors above ground and an occupied basement, $150 \mathrm{~m}^{2}$, occupied by 3 persons, typical older construction.

These 3 houses are all-electric, and hourly metered electrical use was obtained for one full year.

The commercial building is an all-electric 8-story building with $5600 \mathrm{~m}^{2}$ floor area, mainly used for offices, with a relatively good building envelope. Meter data was obtained from a Hydro-Québec case study (2019).

\section{Urban-level building models implemented in UMI}

As described in the introduction, UMI separates the building geometrical information from energy performance parameters such as envelope thermal properties, casual gains densities and schedules, etc.

The geometry of all selected buildings is entered in Rhino with a simplified urban context. The WWR is defined from the information collected for each building.

The differences between successive refinements in UMI models consist of parameters included in the "template" description - in this project, two templates are used, an office building and a residential building. The successive levels of refinement for UMI models are using:

- default parameters, which were obtained for a different local context;

- local building energy code parameters;

- statistical data on the thermal characteristics of buildings at the regional level;

- the calibrated parameters from the detailed models;

- selected adjusted parameters (see below).

UMI Default Model

UMI is developed in Cambridge, MA (USA), so the actual climate zone and urban morphology are different from Montréal, but both are North-American cities in a relatively cold climate (ASHRAE zones 5 and 6 respectively (2013)) so using predefined archetypes would be a reasonable first step for a user trying to model a neighborhood in Montréal. The "B_Off_0" (office building) and "B_Res_0_WoodFrame" (residential building) templates were selected and used unmodified. It should be stressed that this use of default templates in a different context is not recommended by UMI developers, and certainly does not represent good practice - but it is, in the authors point of view, a reasonable approximation of how beginners or non-experts users would get their first results.

UMI templates with data from building energy codes

Building energy codes are often used as a proxy in building stock modeling efforts. In Canada, the national CanmetEnergy research laboratory has adapted prototype buildings for various building energy codes (2019). Energy models for 16 different commercial buildingsthe same as their American counterpart (Deru et al. 2011) _ offer the modeling parameters for this set of commercial building UMI templates. More precisely, the 
modeling assumptions for these models are taken from The 2011 Canadian "National Energy Code for Buildings (NECB)" (NRC-IRC 2011).

On the residential side, modelling assumptions from the IECC prototype buildings (US DOE - Building Energy Codes Program 2012) are used. For their similarity in construction types and equipment performance, the Vermont single-family residence (SFR) were selected as the base for this set of residential templates. Some assumptions were adapted to fit the imposed format of UMI templates: the different internal gains specified in IECC archetypes were summed and their respective schedules averaged by nominal weight.

\section{UMI templates with statistical performance data}

Statistical templates offer a third modeling assumption that do not rely on building energy codes but rather on collected survey data. For residential buildings, the Energuide database (Natural Resources Canada 2018) contains pre- and post-retrofit detailed parameters (e.g. walls and fenestration U-value, etc.) for around 460000 houses across Canada.

The database contains thermal performance characteristics for those houses (infiltration rate, Uvalues, type of HVAC system, number of occupants, etc.), allowing us to compute statistical parameters for this set of UMI templates. This data is classified by housing type in 11 different categories (attached or detached duplex, row house, single detached, etc.). For the purpose of comparing different residential building archetypes, we used this database to create a "weighted average" archetype based in Montreal, QC. A total of 7624 SFR were used to calculate average values for each archetype's properties.One subtlety of the Energuide database is that windows are described by Hot2000 codes referring to a simplified modeling program developed by Natural Resources Canada (2019). These codes consist of 6 digits to define the window (Hawk-Eye n.d.). We selected about 40 prominent window codes, which represent $80 \%$ of the database. The corresponding windows were modeled in WINDOW (LBNL 2013) to obtain the corresponding Uvalues and Solar Heat Gain Coefficients (SHGC), which were then treated as other building parameters to obtain a weighted average value.

\section{UMI with calibrated parameters from detailed models}

As discussed above, the reference for "achievable modeling accuracy" in our study is provided by calibrated detailed models. It seemed logical to transfer the calibrated parameters from the detailed models to the UMI template, in order to improve their accuracy. For parameters controlled by schedules, such as the DHW water draw, equipment and lighting energy use, typical daily, weekly and yearly schedules were created from the detailed models values. Lighting and equipment were lumped into one category in UMI to simplify data input. For the commercial building, a simple cubic geometry was inferred from the non-geometrical DOE-2 description of the building.

\section{UMI model with adjusted parameters}

The results presentation will show that some results obtained with the UMI model using calibrated parameters from the detailed models were in fact worse than other UMI model variants. Canceling errors may be at play both in the UMI models and in the detailed models, so that "fitted" parameters for specific aspects cannot be transferred between models. A simple example would be a model that overestimates the U-value of windows but compensates the extra thermal losses by a reduced infiltration - transferring the calibrated infiltration from this model to another one which would have a more correct window $\mathrm{U}$-value would in fact degrade the performance. In order to still obtain an adjusted (if not fully calibrated) version of the UMI models, a last variant of the archetype templates was developed in which the infiltration rate (a usual target for rough "calibration") was adjusted to match the yearly energy use.

\section{Detailed models}

The detailed reference models represent the best practices in modeling individual buildings, with a level of detail that would not be in line with urban modeling. They were added to the comparison in order to obtain an educated guess of the energy end-use breakdown, and to offer an upper target for the accuracy of urban-level models.

For the commercial (office) building, a detailed SIMEB model was obtained from a Hydro-Québec case study provided with the software (2019). The model is nongeometrical. It has been defined by 3 functional zones: office, corridors and server/computer rooms. SIMEB can use different computational engines, and the DOE-2 engine was selected in this work.

For the residential buildings, individual models were developed in TRNSYS (Klein et al. 2018). The 3D geometry of the models was entered in SketchUp. Base assumptions were used to assign thermal properties in the multizone building model (TRNSYS Type 56). The energy use of plug loads and lighting (Appliances, Lighting and Equipment, ALE in the following) was assigned through calibration as one of the 23 measured profiles published by Johnson and Beausoleil-Morrison (2017). Similarly, one of the 12 measured profiles published by Edwards et al. (2015) was used for the Domestic Hot Water usage.

\section{Model calibration}

Calibration was performed through an iterative trial-anderror process, using engineering judgement to prioritize the parameters to be adjusted and the allowed variations.

The reference data for calibration is hourly building-level electricity use (all buildings are all-electric). Weather files were obtained for the actual year matching the measured data and for the closest weather station from HydroQuébec's SIMEB website (Hydro-Québec 2019). Details on the envelope structure and estimated thermal performance were obtained and matched to typical local construction practices. Typical schedules retrieved form 
the U.S. Department of Energy (2018) were used to define the occupation of the buildings depending on the day of the week and the hour of the day. After going through the previous procedures, we were able to run the simulation and compare the results with the measured data. Finally, as a sanity check on the calibration results, we used the statistical coefficients suggested by the ASHRAE Guideline 14. Table 1 presents the maximum values of the NMBE and CVRMSE we get for residential and commercial sectors, and Table 2 (at the end of the document) summarizes the parameters used in each model.

Table 1: ASHRAE-14 ratio results for building calibration

\begin{tabular}{|c|c|c|c|c|}
\hline & \multicolumn{2}{|c|}{ Residential } & \multicolumn{2}{c|}{ Commercial } \\
\hline & Hourly & Monthly & Hourly & Monthly \\
\hline NMBE & $1.87 \%$ & $1.87 \%$ & $3.61 \%$ & $3.61 \%$ \\
\hline CVRMSE & $59.4 \%$ & $9.06 \%$ & $30.3 \%$ & $6.52 \%$ \\
\hline
\end{tabular}

We notice for the residential stock that the calibration shows some limitations. The hourly CVRMSE does not respect the standard of the ASHRAE Guideline 14, hinting that the good monthly fit may result from canceling errors.

\section{Results and Discussion}

The results discussed below were obtained from the successively refined UMI models and are compared to the detailed (and calibrated) simulation results and to measured energy use.

\section{Annual Energy Consumption}

The following figures (bar graphs) show the annual energy consumption per end-use (except for the measured values for which only the total is known). There is a large variation in energy intensity, from $90 \mathrm{kWh} \mathrm{m}^{-2} \mathrm{y}^{-1}$ to $240 \mathrm{kWh} \mathrm{m}^{-2} \mathrm{y}^{-1}$. Differences related to the geometry (envelope-to-floor area ratio, WWR, external vs. adjacent walls/ceilings) are present but relatively minor, so that the UMI archetypes give similar results for the 3 houses when using the same parameters (compare e.g. the UMI code results for the 3 houses). Populating UMI archetypes with code or statistical values can deliver a reasonable approximation (House 3 for code, House 2 for statistics) but also lead to very large differences.

It is also interesting to note that applying the "best guess" thermal parameters (i.e. calibrated values from the detailed models) to the UMI archetypes can in fact lead to larger errors (see Houses 2 and 3). As discussed above, canceling errors are probably at play in all models, but in different ways. But it is surprising that the differences between the detailed model and the UMI archetype using the same parameters can go from a slight underestimation (House 1) to a very large overestimation (House 3 ) of the heating load.

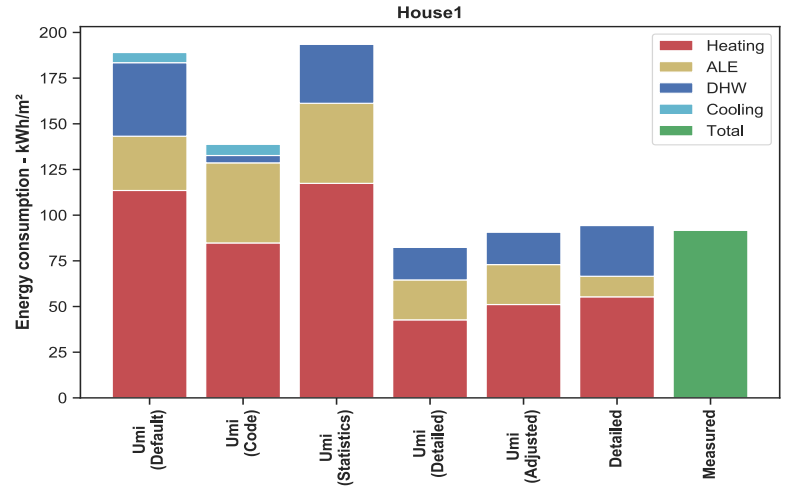

Figure 1 : Total end-use energy consumption per square meter area for residential building (Housel).

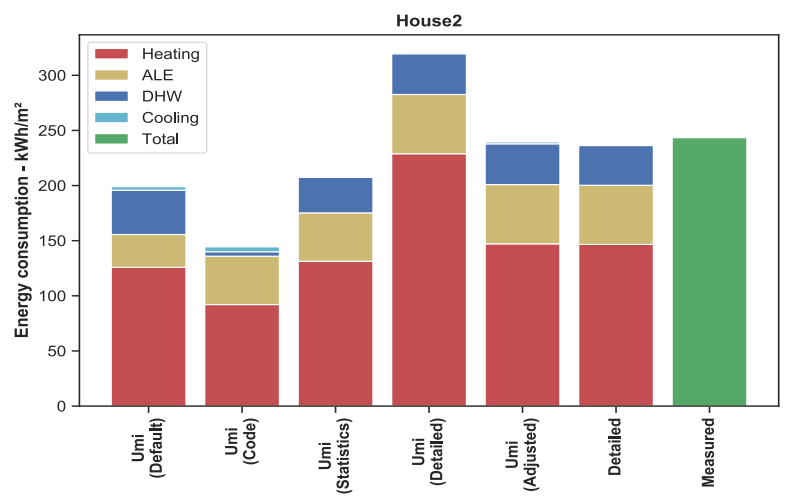

Figure 2 : Total end-use energy consumption per square meter area for residential building (House2).

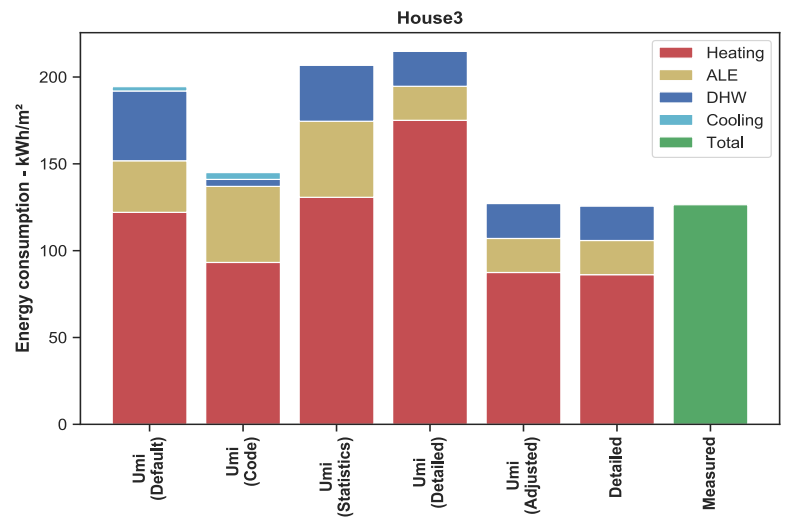

Figure 3 : Total end-use energy consumption per square meter area for residential building (House3).

For the medium office building (Figure 4), the most striking difference between UMI archetypes and the detailed model is the absence of fans and pumps. The UMI approach ignores HVAC systems and applies fixed COPs a posteriori, so degrading the COP could probably lead to a better approximation of the total energy, but the heat / cold / electricity ratio would still be poorly estimated, which is a disadvantage for district energy studies. The impact of fan power on the heating and cooling load is also missed. Because of these aspects, no attempt was made to adjust infiltration in the UMI model to match the total energy use, so there is no "UMI (Adjusted)" model for the office building. 


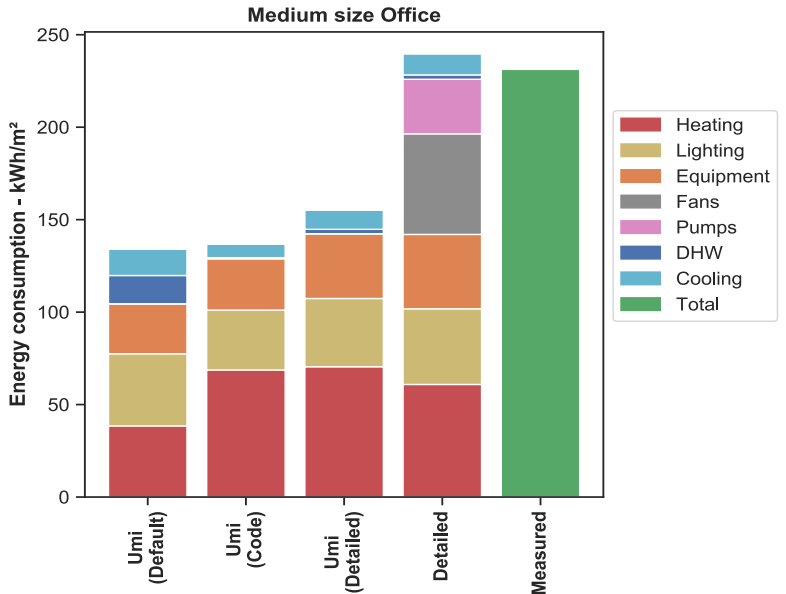

Figure 4 : Total end-use energy consumption per square meter area for a medium-size building office

\section{Daily Energy Use}

Figure 5 shows the daily average energy use for different models, for a selected winter week. The UMI archetypes all show a strong variation between the week days, with a slow decrease during the week. This variation is not apparent in the measured energy use nor in the detailed model. Using adjusted values improves the goodness-offit but does not remove this - apparently incorrect - trend.

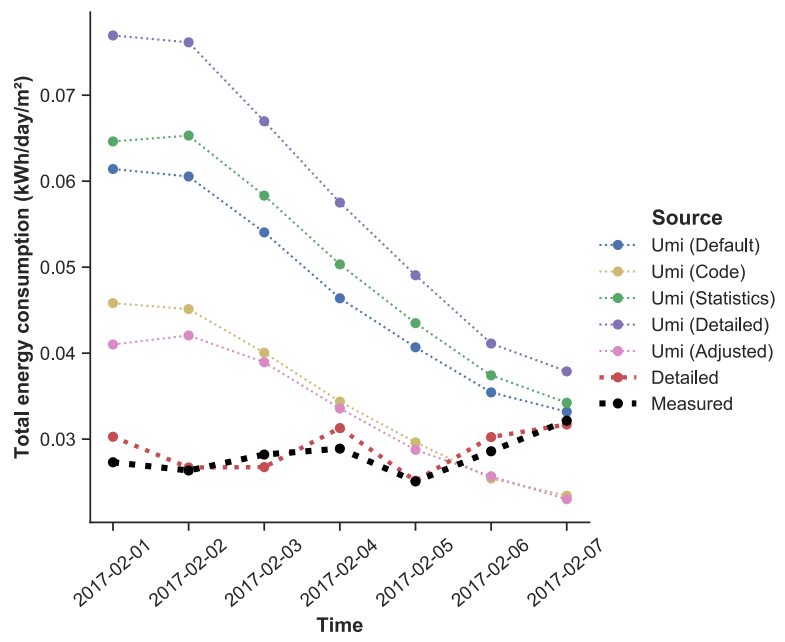

Figure 5 : Average daily energy consumption for one residential building for a winter week.

Figure 6 shows the daily average energy use for the office building during a winter week (Feb 2 and 3 are Saturday and Sunday). Schedules and the missing fans/pumps category (see above) cause large differences between the UMI archetypes and the detailed model.

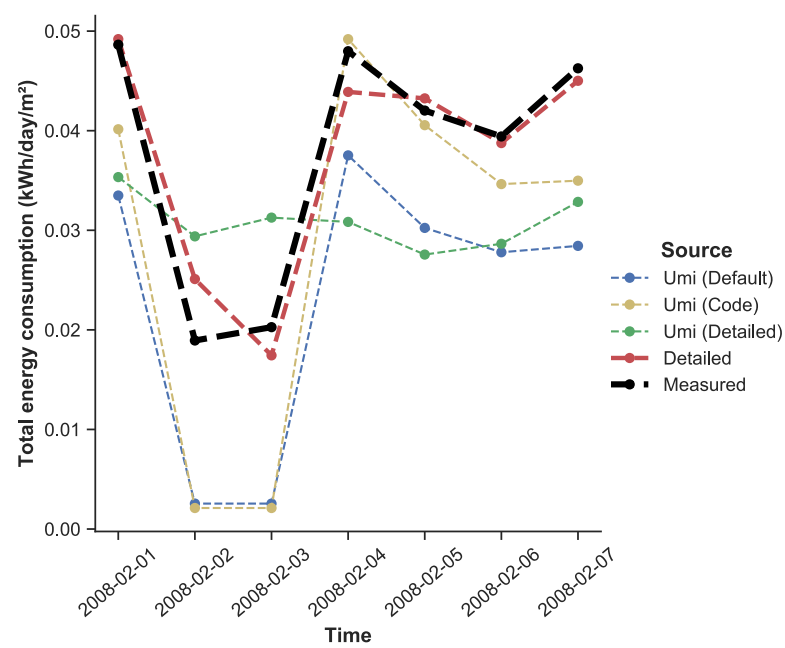

Figure 6 : Average daily energy consumption for one commercial building for a winter week.

\section{Hourly Energy Use}

Figure 8 shows the hourly energy use for a winter day (February $2^{\text {nd }} 2017$ ) in House 3 . The graph shows that UMI archetypes seem to underestimate the dynamic variations of heating load over the day.

Figure 9 shows a winter weekday for the office building. Even though schedules were adapted in the UMI archetypes, the dynamic profile seems largely off, partly because of the missing fans/pumps energy use, but also partly because of an apparent difference of the heating load during occupied hours (increasing in the detailed model, decreasing in the UMI archetype).

\section{Load duration curves}

As mentioned in the introduction, many urban energy modeling studies aim at assessing district energy scenarios. The heat load duration curve, which presents the number of hours that a given load is exceeded, is a useful representation of the dynamic district load to perform optimization studies (Letellier-Duchesne et al. 2018) Figure 7 presents load duration curves of the heat loads (space heating and DHW) for the residential buildings (the 3 houses were aggregated in these curves). The UMI Default, Statistics, and Detailed variants largely overestimate the annual heating energy, so the load duration curve is always above the one for the Detailed model. But adjusting the UMI parameters to match the yearly load seems to lead to a duration curve that underestimates the early part of the duration curve (higher loads) and overestimates the duration of lower loads. This confirms that adjusting the yearly energy use does not guarantee a better estimation of the dynamic energy use, which is important for energy flexibility and district systems studies.

Figure 10 shows the same load duration curve (space heating + DHW) for the office building. In this case the annual heat load is not very far from the results of the Detailed model (see Figure 4), but the load duration curve shows very large deviations, once more showing that a reasonable annual heating load estimate does not guarantee that the dynamic profile will be correctly 
estimated. A striking difference is also apparent in the peak hourly load, which ranges from $73 \mathrm{~W} / \mathrm{m}^{2}$ (Detailed) to $220 \mathrm{~W} / \mathrm{m}^{2}$ (UMI Default). In order to get a reasonable approximation of the hourly peak load at the neighborhood level, diversity would need to be addressed in all cases (including for the Detailed model), and there is no easy way to do this in the current UMI user interface, which focuses on obtaining annual and monthly energy performance indicators. However, one could introduce diversity using two methods, the first manually and the second parametrically. In the first method, users produce template variations by enforcing different parameter ranges. The second method involves the use of the parametrical development plugin, Grasshopper, another Rhinoceros's plugin. The measured data only provides the total energy use, not the share of heating and DHW, so load duration curves were plotted for the total energy use. They are not shown here due to the lack of space, but they show a very good agreement between the Detailed (calibrated) model and the measured data.

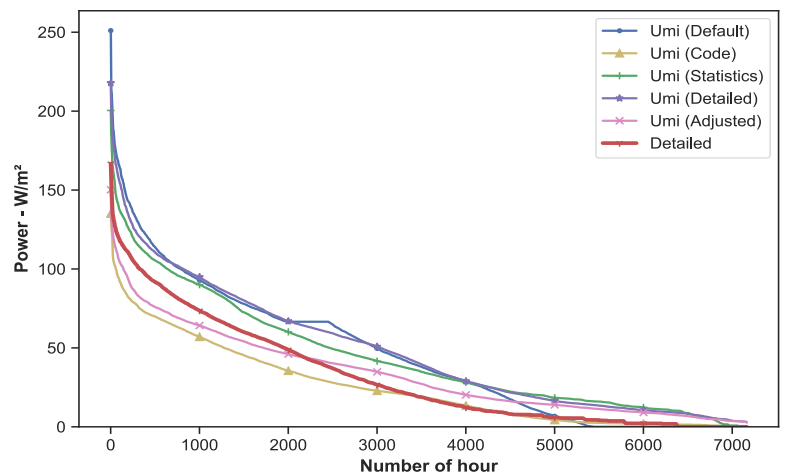

Figure 7 : HEAT (Heating + DHW) load duration curve for the 3 residential buildings (combined)
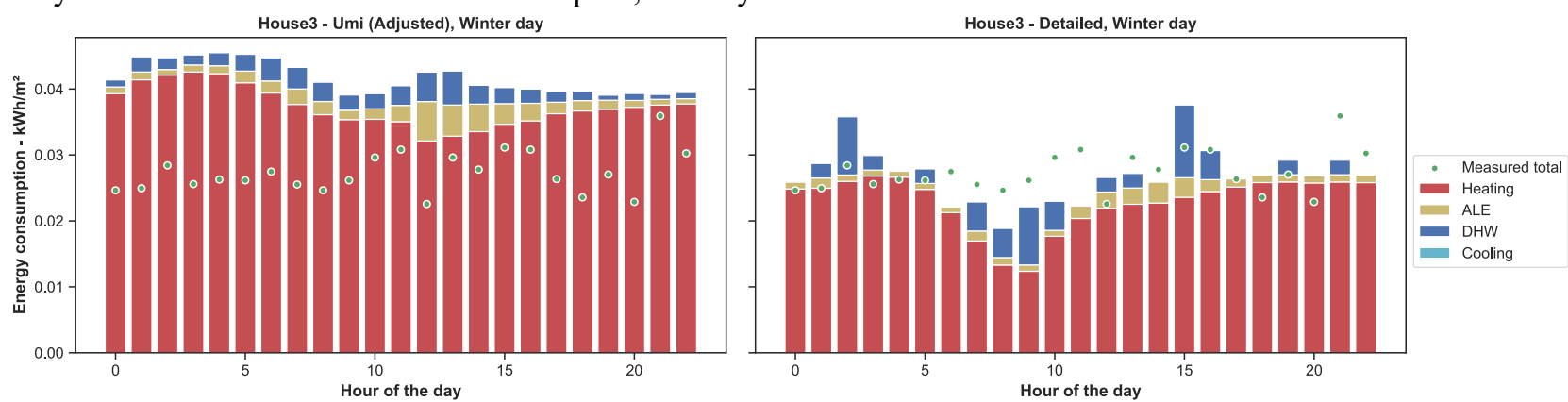

Figure 8 : Hourly energy consumption of a residential building for a winter day
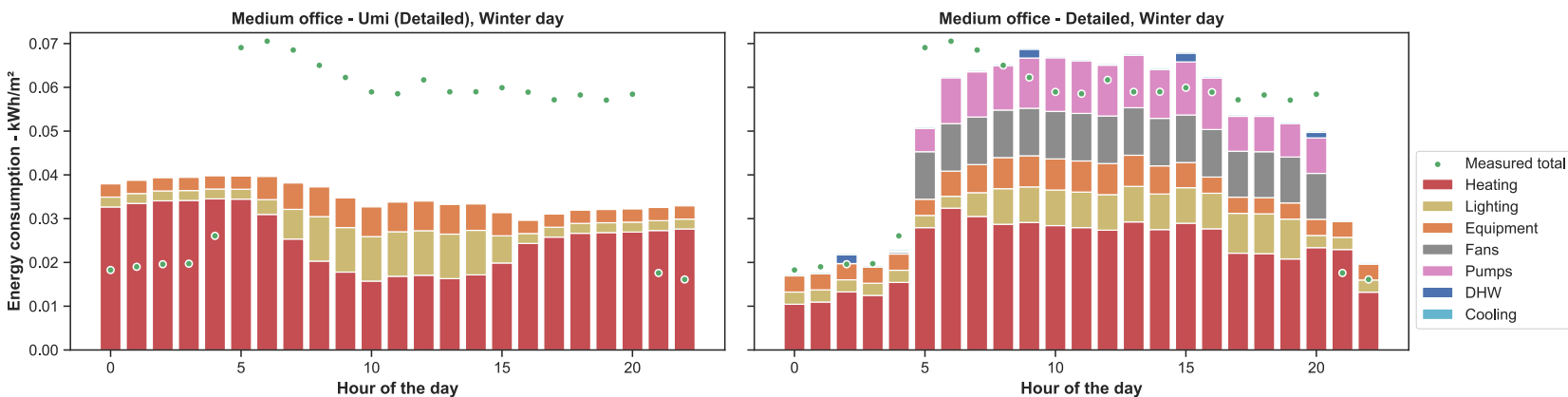

Figure 9 : Hourly energy consumption of a commercial building for a winter day

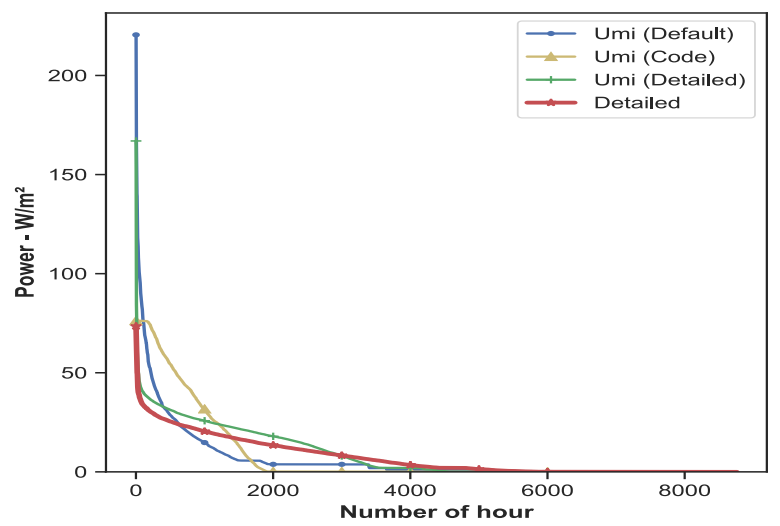

Figure 10 : HEAT (Heating + DHW) load duration curve for a commercial building

\section{Conclusion}

Archetypes used in Urban Building Energy Modeling (UBEM) approaches do not intend to represent individual buildings accurately, but they should provide a reasonable estimate of aggregated energy performance indicators. Their parameters must be adapted to represent the local context, and different data sources are typically used for that purpose: building codes, statistical data, and measured energy use. The hourly energy use profiles of different archetype parametrizations were obtained for selected residential and commercial buildings in UMI, a state-of-the-art UBEM environment. These results were compared to detailed simulation models developed in other simulation tools (TRNSYS and SIMEB using the DOE-2 engine), and to hourly measured data. 
The results show that archetypes using more refined estimates of thermal and building usage parameters do not necessarily deliver better results for the selected buildings, hinting that canceling errors may be at play (both in the urban-level and in the detailed models). This can be problematic when studying building retrofit options (e.g. insulation), because physical parameters might be poorly calibrated. One practical conclusion is that parameters obtained from calibrated detailed models cannot be directly transferred to archetypes.

UMI archetypes used in this work were shown to deliver reasonable annual energy use results, in line with the tool focus, with two caveats. UMI archetypes seem to react differently from detailed models to changes in boundary conditions (external vs. adjacent walls, and contact with the ground). The simplified modeling approach, which focuses on envelope and solar aspects and adopts fixed COP values to represent HVAC systems, leads to the absence of some energy end-uses, such as fans and pumps, which can be significant in commercial buildings. Hourly profiles were assessed by comparing representative days and load duration curves, which are relevant for district energy studies. The hourly results show large differences between the urban-level archetypes and detailed models, indicating that there may be a need to improve the modeling approach if the aim of urban-level analyses evolves from assessing annual indices to assessing energy flexibility and dynamic grid interaction at the neighborhood or city level. Further work should aim at generalizing the results of this study, based on a very small number of buildings, and further investigating differences between detailed and urbanlevel models.

\section{References}

ASHRAE. 2013. Climatic Data for Building Design Standards (Standard 169-2013). Atlanta, GA, USA.

CanmetENERGY. 2019. "Building Technology Assessment Platform (BTAP)."

Cerezo, Carlos, Julia Sokol, Christoph Reinhart, and Adil Al-mumin. 2015. "Three Methods for Characterizing Building Archetypes in Urban Energy Simulation. A Case Study in Kuwait City." Pp. 2873-80 in Proceedings of BS2015: 14th Conference of International Building Performance Simulation Association, Hyderabad, India, Dec. 7-9. Hyderabad, India: International Building Performance Simulation Association.

Deru, Michael, Kristin Field, Daniel Studer, Kyle Benne, Brent Griffith, Paul Torcellini, Bing Liu, Mark Halverson, Dave Winiarski, Michael Rosenberg, Mehry Yazdanian, Joe Huang, and Drury Crawley. 2011. U.S. Department of Energy Commercial Reference Building Models of the National Building Stock. Golden, Colorado.

Edwards, Skai, Ian Beausoleil-Morrison, and André Laperrière. 2015. "Representative Hot Water Draw Profiles at High Temporal Resolution for Simulating the Performance of Solar Thermal Systems." Solar Energy 111:43-52.

Filogamo, Luana, Giorgia Peri, Gianfranco Rizzo, and Antonino Giaccone. 2014. "On the Classification of Large Residential Buildings Stocks by Sample Typologies for Energy Planning Purposes." Applied Energy 135:825-35.

Hawk-Eye. n.d. "HOT2000 Internal Codes for Windows." 5-7.

Heiple, Shem and David J. Sailor. 2008. "Using Building Energy Simulation and Geospatial Modeling Techniques to Determine High Resolution Building Sector Energy Consumption Profiles." Energy and Buildings 40(8):1426-36.

Hydro-Québec. 2019. "Simulation Énergétique Des Bâtiments." Retrieved January 27, 2019 (https://www.simeb.ca/).

Johnson, Geoffrey and Ian Beausoleil-Morrison. 2017. "Electrical-End-Use Data from 23 Houses Sampled Each Minute for Simulating Micro-Generation Systems." Applied Thermal Engineering 114:144956.

Klein, S. A., W. A. Beckman, J. W. Mitchell, J. A. Duffie, N. A. Duffie, T. L. Freeman, J. C. Mitchell, J. E. Braun, B. L. Evans, J. P. Kummer, R. E. Urban, A. Fiksel, J. W. Thornton, N. J. Blair, P. M. Williams, D. E. Bradley, T. P. McDowell, Michaël Kummert, and M. J. Duffy. 2018. "TRNSYS 18 - A TRaNsient SYstem Simulation Program, User Manual. Version 18.0."

Kohler, Niklaus and Uta Hassler. 2002. "The Building Stock as a Research Object." Building Research and Information 30(4):226-36.

LBNL. 2013. THERM 6.3 / WINDOW 6.3 NFRC Simulation Manual. Berkeley, CA, USA: Lawrence Berkeley National Laboratory.

Letellier-Duchesne, Samuel, Shreshth Nagpal, Michaël Kummert, and Christoph Reinhart. 2018. "Balancing Demand and Supply: Linking Neighborhood-Level Building Load Calculations with Detailed District Energy Network Analysis Models.” Energy 150:91325.

Mata, É., A. Sasic Kalagasidis, and F. Johnsson. 2014. "Building-Stock Aggregation through Archetype Buildings: France, Germany, Spain and the UK." Building and Environment 81:270-82.

Natural Resources Canada. 2018. "EnerGuide for Houses." Retrieved September 21, 2018 (https://www.nrcan.gc.ca/energy/efficiency/homes/1 6654).

Natural Resources Canada. 2019. "Tools for Industry Professionals (HOT2000)." Retrieved January 31, 2019 (https://www.nrcan.gc.ca/energy/efficiency/homes/2 0596). 
NRC-IRC. 2011. National Energy Code for Buildings 2011. Ottawa, ON, CAN.

Reinhart, Christoph F. and Carlos Cerezo Davila. 2016. "Urban Building Energy Modeling - A Review of a Nascent Field." Building and Environment 97:196202.

Reinhart, Christophe. 2019. "Urban Modeling Interface." Sustainable Design Lab. Retrieved January 27, 2019 (http://urbanmodellinginterface.ning.com/).

Robert McNeel \& Associates. 2019. "Rhinoceros." Retrieved January 27, 2019 (https://www.rhino3d.com/\#).

Samuelson, Holly W., Arash Ghorayshi, and Christoph F. Reinhart. 2015. "Analysis of a Simplified Calibration Procedure for 18 Design-Phase Building Energy Models." Journal of Building Performance Simulation 9(1):17-29.

Sokol, Julia, Carlos Cerezo Davila, and Christoph Reinhart. 2017. "Validation of a Bayesian-Based Method for Defining Residential Archetypes in Urban Building Energy Models." Energy and Buildings 134:11-24.

U.S. Department of Energy's Building Technologies Office. 2019. "EnergyPlus." Retrieved January 27, 2019 (https://energyplus.net/).

US Department of Energy. 2018. House Simulation Protocols Report. Washington, DC, USA.

US DOE - BTO. 2018. "Commercial Prototype Building Models." Retrieved August 29, 2018 (https://www.energycodes.gov/development/commer cial/prototype_models).

US DOE - Building Energy Codes Program. 2012. "Residential Prototype Building Models." US Department of Energy. 


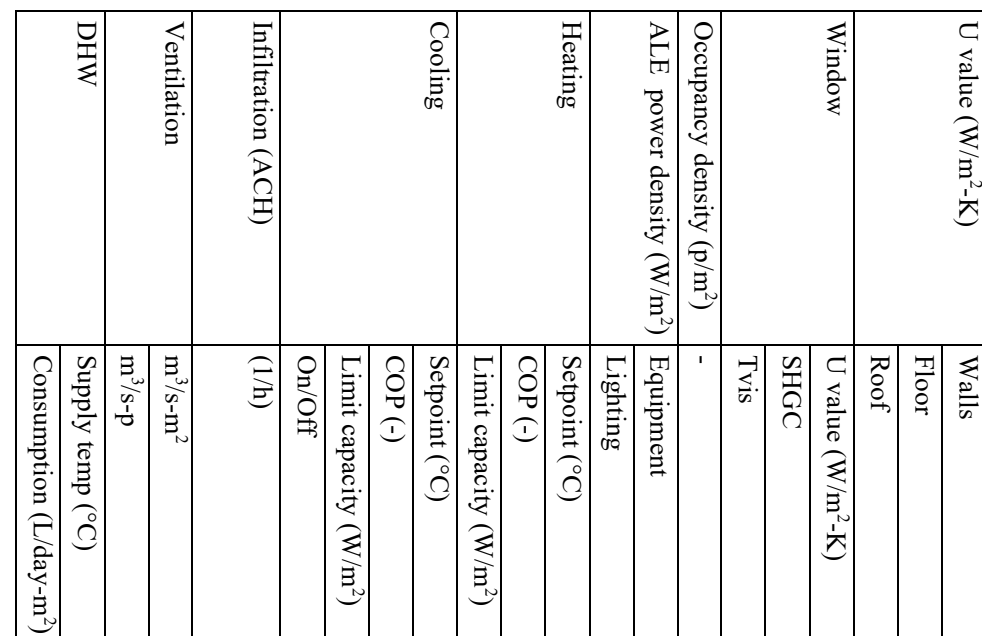

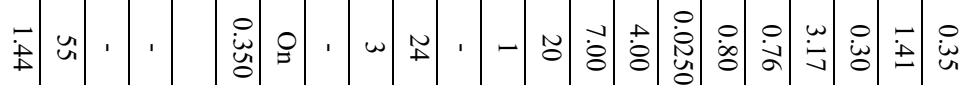

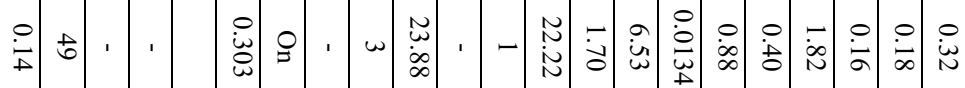

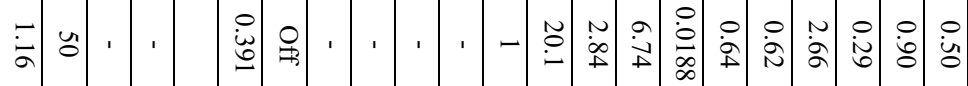

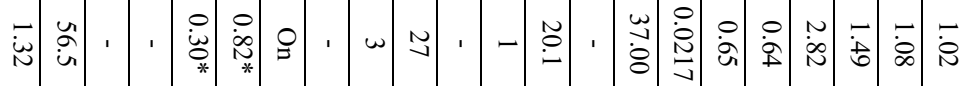

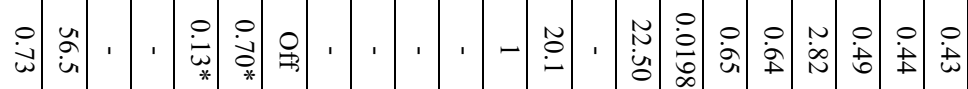

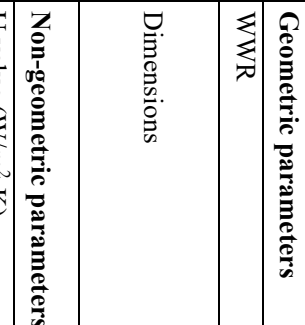

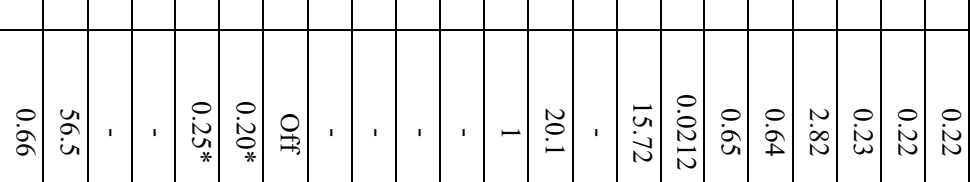

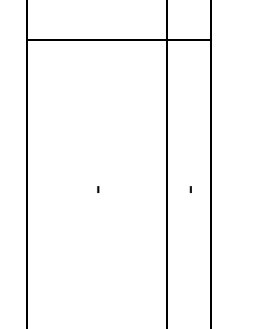

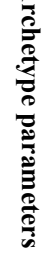

$\int \frac{a}{3}$ 